\title{
Type and amount of available past instances in concept learning
}

DANIEL D. BLAINE, J. L. DUNHAM, AND THOMAS W. PYLE, LABORATORY OF COMPUTER-ASSISTED INSTRUCTION, THE UNIVERSITY OF TEXAS AT AUSTIN

Fifty-six high school Ss classified as high or low in associative memory (AM) participated in a study investigating the effect of two types of available past instances upon performance in a four-category concept problem. Instances which $S$ had classified incorrectly provided either a record of S's own classifications (SR) or a record of correct classifications (CR) at four levels of availability. The results indicated that performance was facilitated only in conditions in which a CR was available. Optimal performance of Ss low in $A M$ was achieved at a low level of $C R$ availability while $S s$ high in $A M$ continued to improve as $C R$ availability increased.

Several authors (e.g., Bourne, Goldstein, \& Link, 1964; Cahill \& Hovland, 1960; Pishkin, 1967; Pishkin \& Wolfgang, 1965; and Pishkin, Wolfgang, \& Rasmussen, 1967) have demonstrated the facilitative effect of available past instances in concept learning. This facilitative effect has typically been interpreted as a reduction in the memory requirements associated with a concept learning task. Beyond this interpretation, few attempts have been made to determine the aspects of available past instances which are important to the solution of a concept task.

In one attempt, Pishkin \& Wolfgang (1965) employed a four-category problem with which they varied the type of instances available. In one group, instances which Ss classified correctly were available in the correct category. In a second group, instances which had been classified incorrectly were available in the incorrect category which $S$ had chosen. In the third group both types of instances were available. In the groups where the available instances provided $S$ with a correct record (CR) of classification, availability was facilitative. In the second group $S$ was provided with a record of his own incorrect responses (SR), and availability did not facilitate performance. Thus, availability was facilitative only when Ss had instances available which they had classified correctly.

This suggests that the facilitative effect of availability is due, at least in part, to providing Ss with a record of instances and their correct classification. Therefore, in the present study it was predicted that availability would be facilitative when it provided S with a CR, even when $S$ originally responded incorrectly to the instances now available. In addition, the availability of such a CR was expected to reduce the memory requirement in the concept task, since under these conditions Ss would not be forced to recall past instances and their correct classifications. Consequently, it was predicted that Ss more capable of recalling the correct classification of past instances, i.e., high in associative memory, would profit less from the availability of a CR. It was also predicted that Ss low in associative memory would profit more from an increase in the number of instances involved in the CR. Subjects

The Ss were 56 students from an Austin, Texas high school. Design

The design was a 2 by 2 by 3 factorial corresponding to two levels of Memory: High (H) vs Low (L); two Types of availability: incorrectly classified instances available in the correct category (CR) vs incorrectly classified instances available in the incorrect category (SR); and three Amounts of availability: 1, 2, or 4 instances per category. In addition, a control group was run in which zero instances were available.

Task and Procedure

Prior to the concept learning task, a short-term memory for associations test (Ma-1, Picture-Number Test from French, Ekstrom, \& Price, 1963) was administered to Ss in a group setting. The distribution of scores was dichotomized at the median in order to assign Ss randomly to conditions on the basis of this memory score.

The concept learning task itself was given to Ss individually. The task was a four-category, two-dimensional conjunctive concept problem. The stimuli were on $3 \times 3$ in. white cards and consisted of six binary dimensions: (a) number of figures, 1 or 2; (b) size of figures, large or small; (c) color of figures, black or white; (d) shape of figures, circle or square; (e) number of borders, 1 or 2 ; and (f) type of borders, solid or broken.

Each $\mathrm{S}$ sat on the opposite side of a table from $E$ with a $22 \times 28$ in. white board divided into four columns (labeled A, B, C, and D) on the table facing $S$. It was explained to $S$ that each of the cards could be classified as an A, B, C, or D on the basis of two of the dimensions. To familiarize $S$ s with the task and the procedure, an example problem was given in which they learned to classify instances after being informed which dimensions were relevant (number and type of borders). All Ss received the example problem under the zero availability condition. Each $S$ was then given additional instructions appropriate to the condition to which he was assigned. As in the example, $S$ was given the deck of 64 cards which he looked at one at a time, telling E to which category he thought the card belonged. After responding $S$ was told whether he was right or wrong and, if wrong, to which category the card belonged. $S$ had an unlimited response interval on each trial. Depending upon the experimental condition, the cards to which $S$ responded incorrectly were placed in the correct category (CR) or the incorrect category (SR) chosen by $\mathrm{S}$ with either 1,2 , or 4 instances allowed to accumulate in each category. When the specified number in a category was exceeded, the earliest instance in that category was removed. Instances which $S$ classified correctly were placed face down to the side of the board. In the zero availability group all instances were placed face down in the same manner. S's responding was terminated after 64 trials or when a preset criterion of 12 consecutive correct responses was achieved.

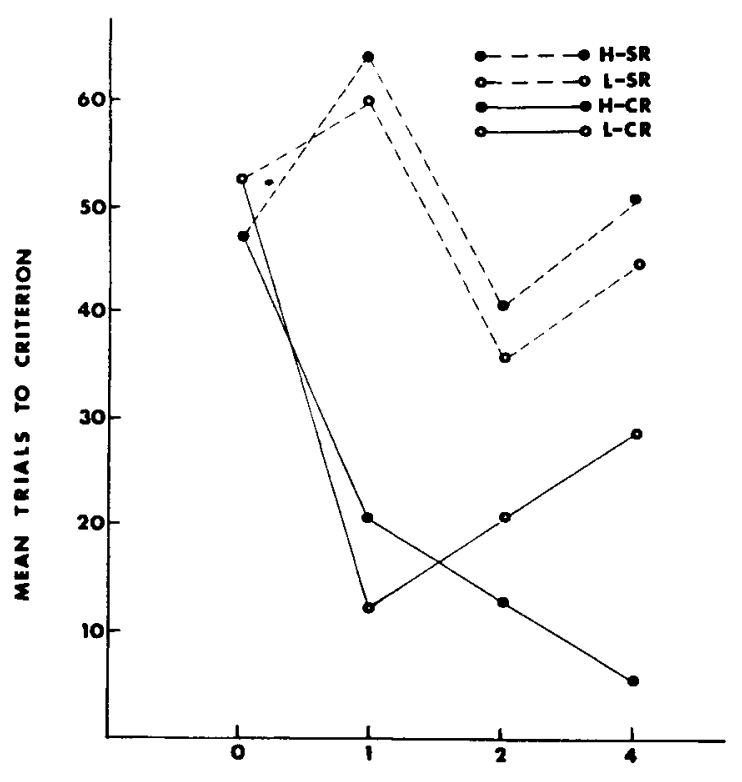

NO. Instances aVAllable pen categorr

Fig. 1. Mean trials-to-criterion for all conditions of Memory, Type of availability, and Amount of availability. 


\section{Results}

The mean trials-to-criterion for each of the conditions are plotted in Fig. 1. A 2 by 2 by 3 analysis of variance (Memory by Type by Amount) was computed with trials-to-criterion as the dependent measure. The main effect of Type was significant $(\mathrm{F}=56.90, \mathrm{df}=1 / 36, \mathrm{p}<.001)$ with $\mathrm{CR}$ availability performance superior to SR performance. The Amount effect and the Type by Amount interaction were also significant $(F=3.28$, $\mathrm{df}=2 / 36$, $\mathrm{p}<.05 ; \mathrm{F}=3.49$, df $=2 / 36, \mathrm{p}<.05)$. Availability was most facilitative when two instances were available per category. Both this effect and the Type by Amount interaction are due to the drop in trials-to-criterion for the SR-2 group.

To test the prediction that CR availability would facilitate Ss low in memory to a greater extent than Ss high in memory, a comparison was made between the $\mathrm{H}$ and $\mathrm{L}$ groups in zero and CR-1 availability. The Memory by Type interaction for these two groups was not significant $(\mathrm{F}=1.18, \mathrm{df}=1 / 12, \mathrm{p}=.30)$.

An analysis of the effect of Memory and Amount within the CR condition yielded a significant main effect for Memory $(F=4.56$, $\mathrm{df}=1 / 18, \mathrm{p}<.05)$ and a significant Memory by Amount interaction $(F=6.20, \mathrm{df}=2 / 18, \mathrm{p}<.01)$.

In addition to the above analyses, a weighted means comparison between zero availability and the $C R$ groups was significant $(\mathrm{F}=27.68, \mathrm{df}=1 / 28, \mathrm{p}<.001)$. A similar comparison between zero availability and the $S R$ groups was not significant $(F=0.003$, $\mathrm{df}=1 / 28, \mathrm{p}=.95$ ).

Discussion

The results indicate that the availability of an SR did not facilitate performance while the availability of a CR was highly facilitative. This suggests that, for available instances to be facilitative, it is necessary for them to be available in such a way that the $S$ can readily associate the correct category with the appropriate instance. Being able to associate instances with the correct category could be facilitative for two reasons. First, in determining the relevant dimensions, $\mathrm{S}$ can gain information about the possible relevancy of dimensions by comparing two or more instances for which the correct classification is known. With the availability of a $C R, S$ is provided with this basis of comparison while with an SR he is not. Second, once the relevant dimensions have been determined by $S$, a $C R$ specifies the combination of values on the relevant dimensions which belong in a particular category. Again, an SR does not provide such information. Ss with an SR available were forced to rely upon memory for the correct classification of the available instances and the information derived from past instances.

Pishkin (1967) found that when Ss were informed of the correct classification of error instances, availability did not have a facilitative effect. Feedback correction as employed by Pishkin (1967) was given under all conditions in the present study, and a facilitative effect due to the availability of a CR was still found. The task employed by Pishkin (1967) was a four-category problem with one four-valued dimension relevant (Wisconsin Card Sorting Test). In the present study, a four-category problem was also employed; however, two binary dimensions were relevant. Bourne et al (1964) found that availability was less facilitative in less complex tasks, i.e., when fewer dimensions were relevant. This would account for the lack of an availability effect found by Pishkin (1967).

The hypothesis that low memory Ss would profit more from the availability of a CR was not confirmed. The indication is that the introduction of CR-1 availability is only slightly more facilitative for low memory Ss than for high memory Ss. Such availability is highly facilitative for both memory groups.

Within the CR condition it was predicted that Ss low in memory would profit more from increasing availability than Ss high in memory. This was not confirmed. The fact that the interaction reached significance in the direction opposite to that predicted indicated that Ss high in memory profited more from increasing availability than did Ss low in memory. Ss low in memory achieved optimal performance at one instance per category available with a decrease in the facilitative effect of availability at levels above one. Bourne et al (1964) interpreted a similar effect as due to a lack of time to process the available information. When the $S$ s were given an unlimited response interval, performance continued to improve with increasing availability. Since an unlimited response interval was afforded all Ss in the present study, the optimal level of low memory Ss cannot be interpreted as due to lack of time to process the available information. The mean number of trials-to-criterion for Ss high in memory continued to decrease as the limit on the number of instances available within a given category increased, suggesting that Ss scoring high on the memory test could utilize the information efficiently while low memory Ss were unable to do so. Thus, the measure of associative memory employed here may also be an indication of the ability to utilize information which is available as well as the ability to recall previously presented information.

It was concluded that for availability to be facilitative, it must provide $S$ with information relevant to the solution of the task, and given that such information is available, there is an optimal level of availability of such information which is, at least in part, a function of S's ability to utilize it efficiently.

\section{REFERENCES}

BOURNE, L. E., Jr., GOLDSTEIN, S., \& LINK, W. E. Concept learning as a function of availability of previously presented information. $J$. exp. Psychol, 1964, 67, 439-448.

CAHILL, H. E., \& HOVLAND, C. I. The role of memory in the acquisition of concepts. J. exp. Psychol, 1960, 59, 137-144.

FRENCH, J. W., EKSTROM, R. B., \& PRICE, L. A. Manual for kit of reference tests for cognitive factors. Princeton, N.J.: Educational Testing Service, 1963.

PISHKIN, V. Supplementary report: Availability of feedback-corrected error instances in concept learning. J. exp. Psychol, 1967, 73, 318-319.

PISHKIN, V., \& WOLFGANG, A. Number and type of available instances in concept learning. J. exp. Psychol, 1965, 69, 5-8.

PISHKIN, V., WOLFGANG, A., \& RASMUSSEN, E. Age, sex, amount, and type of memory information in concept learning. J. exp. Psychol., 1967, $73,121-124$. 